\title{
Periodic Review Probabilistic Multi-Item Inventory System with Zero Lead Time under Constraints and Varying Order Cost
}

\author{
Hala A. Fergany \\ Lecturer of Mathematical Statistics, Faculty of Science, Tanta University, Tanta, Egypt
}

\begin{abstract}
This study treats the probabilistic safety stock n-items inventory system having varying order cost and zero lead-time subject to two linear constraints. The expected total cost is composed of three components: the average purchase cost; the expected order cost and the expected holding cost. The policy variables in this model are the number of periods $\mathrm{N}_{\mathrm{r}}{ }^{*}$ and the optimal maximum inventory level $\mathrm{Q}_{\mathrm{mr}}{ }^{*}$ and the minimum expected total cost. We can obtain the optimal values of these policy variables by using the geometric programming approach. A special case is deduced and an illustrative numerical example is added.
\end{abstract}

Key words: Probabilistic model, zero lead-time, safety stock, multi-item, varying order cost, geometric programming

\section{INTRODUCTION}

In many situations demand is probabilistic since it is a random variable having a known probability distribution. All researchers have studied unconstrained probabilistic inventory models assuming the ordering cost to be constant and independent of the number of periods. Hadley, et $a l^{[4]}$ and Taha ${ }^{[6]}$, has examined unconstrained probabilistic inventory problems.

Fabric and Banks ${ }^{[3]}$ studied the probabilistic singleitem, the single source inventory system with zero leadtime, using the classical optimization. Also Hariri and Abou-El-Ata ${ }^{[5]}$ deduced the deterministic multi-item production lot size inventory model with a varying order cost under a restriction: a geometric programming approach. Recently Abou-El-Ata, et al ${ }^{[1]}$ studied the probabilistic multi-item inventory model with varying order cost under two restrictions: a geometric programming approach.

The aim of this study is to investigate the probable safety stock multi-item, single source inventory model with zero lead-time and varying order cost under two constraints, one of them of the expected holding cost and the other on the expected cost of safety stock. The optimal amount of periods $\mathrm{N}_{\mathrm{r}}^{*}$, the optimal maximum inventory levels $\mathrm{Q}^{*}{ }_{\mathrm{mr}}$ and min $\mathrm{E}$ (TC) are obtained. Also special case is deduced and an illustrative numerical example is added.

Model development: The following notations are adopted for developing our model:

$\mathrm{C}_{\mathrm{pr}} \quad=$ The purchase cost of the $\mathrm{r}^{\text {th }}$ item,
$\mathrm{C}_{\mathrm{or}}\left(\mathrm{N}_{\mathrm{r}}\right)=$ The varying order cost of the $\mathrm{r}^{\text {th }}$ item per cycle

$\mathrm{C}_{\mathrm{hr}} \quad=$ The holding cost of the $\mathrm{r}^{\text {th }}$ item per period

$\overline{\mathrm{I}_{\mathrm{r}}} \quad=$ The expected level of inventory held per $\mathrm{r}^{\text {th }}$ cycle

$\mathrm{x}_{\mathrm{r}} \quad=\mathrm{A}$ random variable represent the demand of the $\mathrm{r}^{\text {th }}$ item during the cycle

$\mathrm{F}\left(\mathrm{x}_{\mathrm{r}}\right)=$ The probability density function of the demand $\mathrm{x}_{\mathrm{r}}$

$\mathrm{E}\left(\mathrm{x}_{\mathrm{r}}\right)=$ The expected value of the demand $\mathrm{x}_{\mathrm{r}}$ $\int_{x_{\mathrm{x}}}^{\mathrm{x}_{\mathrm{m}}} \mathrm{x}_{\mathrm{r}} \mathrm{f}\left(\mathrm{x}_{\mathrm{r}}\right) \mathrm{d} \mathrm{x}_{\mathrm{r}}$, where $\mathrm{x}_{\mathrm{ur}}$ and $\mathrm{x}_{\mathrm{lr}}$ are the maximum value and minimum value of $x_{r}$

$D_{\mathrm{r}} \quad=$ The annual demand rate of the $\mathrm{r}^{\text {th }}$ item per period

$\mathrm{E}\left(\mathrm{D}_{\mathrm{r}}\right) \quad=$ The expected annual demand $\mathrm{D}_{\mathrm{r}}$

$\mathrm{Q}_{\mathrm{mr}} \quad=$ The maximum inventory level of the $\mathrm{r}^{\text {th }}$ item

$\mathrm{N}_{\mathrm{r}} \quad=$ The number of periods, cycle, of the $\mathrm{r}^{\text {th }}$ item (a decision variable) and a review of the stock level of the $\mathrm{r}^{\text {th }}$ item is made every $\mathrm{N}_{\mathrm{r}}$ period

$v \quad=$ The positive value representing a part of time for safety stock

$\mathrm{K}_{1} \quad=$ The limitation on the expected holding cost

$\mathrm{K}_{2}=$ The limitation on the expected safety stock cost

$\mathrm{E}(\mathrm{TC})=$ The expected total cost function.

The model analysis: Consider an inventory process in which a review of the stock level is made every $\mathrm{N}_{\mathrm{r}}$ period, $\mathrm{r}=1,2, \ldots, \mathrm{n}$. An amount is ordered so that the stock level has returned to its initial position designated by: $\mathrm{Q}_{\mathrm{mr}}, \mathrm{r}=1,2, \ldots, \mathrm{n}$. To avoid shortage during $\mathrm{N}_{\mathrm{r}}$.

Corresponding Author: Hala A. Fergany, Lecturer of Mathematical Statistics, Faculty of Science, Tanta University, Tanta, Egypt 


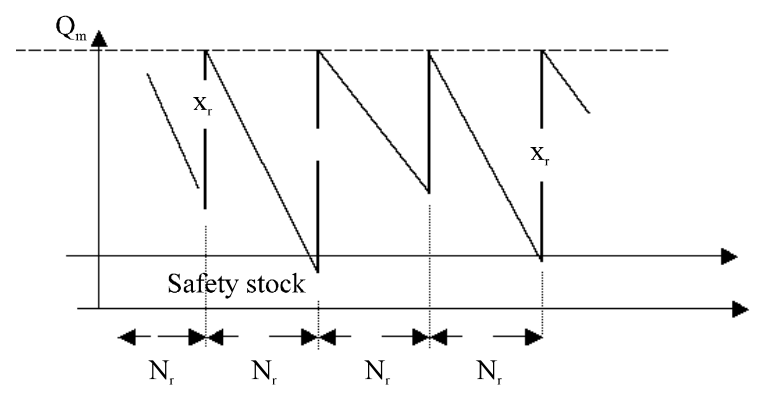

Fig. 1: Inventory system with safety stock

Periods we must maintain a safety stock absorbing demand fluctuation. Also, this is done maintaining the quantity $\mathrm{Q}_{\mathrm{mr}}=\mathrm{x}_{\mathrm{ur}}$ for any cycle $\mathrm{N}_{\mathrm{r}}$. Hence the resulting safety stock, $\mathrm{D}_{\mathrm{r}} \mathrm{v}$, meets the exceed demands cycle $\mathrm{N}_{\mathrm{r}}$. The periodic inventory system is exhibited graphically as shown in Fig. 1.

The expected annual total cost is composed of three components: the expected purchase cost the expected order cost and the expected holding costs as follows:

$$
\begin{aligned}
& \mathrm{E}(\mathrm{TC})=\mathrm{E}(\mathrm{PC})+\mathrm{E}(\mathrm{OC})+\mathrm{E}(\mathrm{HC}), \\
& \mathrm{E}(\mathrm{PC})=\sum_{\mathrm{r}=1}^{\mathrm{n}} \mathrm{C}_{\mathrm{pr}} \mathrm{E}\left(\mathrm{D}_{\mathrm{r}}\right), \mathrm{E}(\mathrm{OC})=\sum_{\mathrm{r}=1}^{\mathrm{n}} \frac{\mathrm{C}_{\mathrm{or}}\left(\mathrm{N}_{\mathrm{r}}\right)}{\mathrm{N}_{\mathrm{r}}},
\end{aligned}
$$

And:

$$
\mathrm{E}(\mathrm{HC})=\sum_{\mathrm{r}=1}^{\mathrm{n}} \frac{\mathrm{C}_{\mathrm{hr}} \overline{\mathrm{I}_{\mathrm{r}}}}{\mathrm{N}_{\mathrm{r}}}
$$

Where:

$$
\overline{\mathrm{I}_{\mathrm{r}}}=\mathrm{N}_{\mathrm{r}}\left[\mathrm{Q}_{\mathrm{mr}}-\frac{\mathrm{E}\left(\mathrm{x}_{\mathrm{r}}\right)}{2}\right]
$$

And:

$$
\mathrm{E}\left(\mathrm{x}_{\mathrm{r}}\right)=\mathrm{E}\left(\mathrm{D}_{\mathrm{r}}\right) \mathrm{N}_{\mathrm{r}}
$$

Then:

$$
\mathrm{E}(\mathrm{HC})=\sum_{\mathrm{r}=1}^{\mathrm{n}} \frac{\mathrm{C}_{\mathrm{hr}}\left[2 \mathrm{Q}_{\mathrm{mr}}-\mathrm{E}\left(\mathrm{D}_{\mathrm{r}}\right) \mathrm{N}_{\mathrm{r}}\right]}{2}
$$

The Optimization of the decision variables $\mathrm{N}_{\mathrm{r}}$ and $\mathrm{Q}_{\mathrm{mr}}$ can be performed if we assume that the maximum demand during the cycle, $\mathrm{x}_{\mathrm{ur}}$, is related to the expected demand during the cycle as:

$$
\mathrm{x}_{\mathrm{ur}}=\mathrm{E}\left(\mathrm{x}_{\mathrm{r}}\right) \mathrm{g}\left(\mathrm{N}_{\mathrm{r}}\right)=\mathrm{E}\left(\mathrm{D}_{\mathrm{r}}\right) \mathrm{N}_{\mathrm{r}} \mathrm{g}\left(\mathrm{N}_{\mathrm{r}}\right)
$$

where, $g(\mathrm{~N})$ is a relational function which consider to be:

$$
g\left(N_{r}\right)=\left(\frac{N_{r}+v}{N_{r}}\right)
$$

Hence, the following form gives the expected holding cost per period:

$$
\mathrm{E}(\mathrm{HC})=\sum_{\mathrm{r}=1}^{\mathrm{n}} \frac{\mathrm{C}_{\mathrm{hr}} \mathrm{E}\left(\mathrm{D}_{\mathrm{r}}\right)\left[\mathrm{N}_{\mathrm{r}}+2 v\right]}{2}
$$

The order cost per unit is a varying function of the expected number of periods, $\mathrm{N}_{\mathrm{r}}$, which takes the following form:

$\mathrm{C}_{\mathrm{Or}}\left(\mathrm{N}_{\mathrm{r}}\right)=\mathrm{C}_{\mathrm{or}} \mathrm{N}_{\mathrm{r}}^{\beta}$,

where, $\mathrm{C}_{\mathrm{or}}>0$ and $0.5 \leq \beta<2$ are constants real numbers selected to provide us the best estimation of the cost function.

Our objective is to minimize the relevant expected annual total cost function, according to the previous assumptions of the model:

$$
E(T C)=\sum_{r=1}^{n}\left[\begin{array}{l}
C_{p r} E\left(D_{r}\right)+C_{o r} N_{r}^{\beta-1}+ \\
\frac{C_{h r} E\left(D_{r}\right) N_{r}}{2}+C_{h r} E\left(D_{r}\right) v
\end{array}\right]
$$

i.e.

Under the following constraints:

$$
\left.\begin{array}{l}
\sum_{\mathrm{r}=1}^{\mathrm{n}} \frac{\mathrm{C}_{\mathrm{hr}} \mathrm{E}\left(\mathrm{D}_{\mathrm{r}}\right) \mathrm{N}_{\mathrm{r}}}{2} \leq \mathrm{K}_{1} \\
\sum_{\mathrm{r}=1}^{\mathrm{n}} \mathrm{C}_{\mathrm{hr}} \mathrm{E}\left(\mathrm{D}_{\mathrm{r}}\right) v \leq \mathrm{K}_{2}
\end{array}\right\}
$$

The cost of safety stock insurance is given by the last term in the equation (1), in the safety process an amount is held in excess of the expected requirement as insurance against the risk of a stakeout. The terms $\sum_{r=1}^{n} C_{p r} E\left(D_{r}\right)$ and $\sum_{r=1}^{n} C_{h r} E\left(D_{r}\right) v$ can be posted without any effect. Then the minimum expected total cost can be written as:

$$
\min \mathrm{E}(\mathrm{TC})=\sum_{\mathrm{r}=1}^{\mathrm{n}}\left[\mathrm{C}_{\mathrm{or}} \mathrm{N}_{\mathrm{r}}^{\beta-1}+\frac{\mathrm{C}_{\mathrm{hr}} \mathrm{E}\left(\mathrm{D}_{\mathrm{r}}\right) \mathrm{N}_{\mathrm{r}}}{2}\right]
$$

\section{Subject to:}

$$
\sum_{\mathrm{r}=1}^{\mathrm{n}} \frac{\mathrm{C}_{\mathrm{hr}} \mathrm{E}\left(\mathrm{D}_{\mathrm{r}}\right) \mathrm{N}_{\mathrm{r}}}{2 \mathrm{~K}_{1}} \leq 1 \quad \text { and } \sum_{\mathrm{r}=1}^{\mathrm{n}} \frac{\mathrm{C}_{\mathrm{hr}} \mathrm{E}\left(\mathrm{x}_{\mathrm{r}}\right) \mathrm{U}}{\mathrm{N}_{\mathrm{r}} \mathrm{K}_{2}} \leq 1
$$


Applying the geometric programming techniques to the equation (3) and (4), the enlarged predual function could be written in the following form:

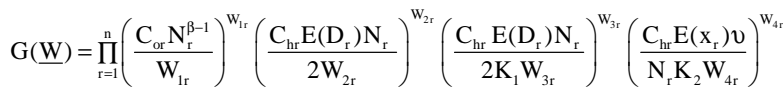

$$
\begin{aligned}
& =\prod_{r=1}^{n}\left(\frac{C_{o r}}{W_{1 r}}\right)^{W_{r t}}\left(\frac{C_{h r} E\left(D_{r}\right)}{2 W_{2 r}}\right)^{W_{2 r}}\left(\frac{C_{h r} E\left(D_{r}\right)}{2 K_{1} W_{3 r}}\right)^{W_{s r}}
\end{aligned}
$$

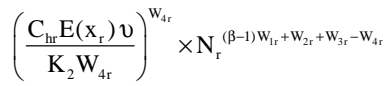

where, $\underline{\mathrm{W}}=\mathrm{W}_{\mathrm{ir}}, 0<\mathrm{W}_{\mathrm{ir}}<1, \mathrm{j}=1,2,3,4, \mathrm{r}=1,2, \ldots, \mathrm{n}$ are the weights and can be chosen to yield the normal and the orthogonality conditions as follows:

$$
\begin{aligned}
& \mathrm{W}_{1 \mathrm{r}}+\mathrm{W}_{2 \mathrm{r}}=1 \\
& (\beta-1) \mathrm{W}_{1 \mathrm{r}}+\mathrm{W}_{2 \mathrm{r}}+\mathrm{W}_{3 \mathrm{r}}-\mathrm{W}_{4 \mathrm{r}}=0, \mathrm{r}=1,2, \ldots, \mathrm{n} .
\end{aligned}
$$

Solving the above equations, we get:

$\mathrm{W}_{\mathrm{lr}}=\frac{1+\mathrm{W}_{3 \mathrm{r}}-\mathrm{W}_{4 \mathrm{r}}}{2-\beta}$ and $\mathrm{W}_{2 \mathrm{r}}=\frac{1-\beta-\mathrm{W}_{3 \mathrm{r}}+\mathrm{W}_{4 \mathrm{r}}}{2-\beta}, \mathrm{r}=1,2, \ldots, \mathrm{n}$.

Substituting from (6) into (5), the dual function is given in the form:

$$
\begin{aligned}
& \mathrm{g}\left(\mathrm{W}_{3 \mathrm{r}}, \mathrm{W}_{4 \mathrm{r}}\right)=\prod_{\mathrm{r}=1}^{\mathrm{n}}\left(\frac{(2-\beta) \mathrm{C}_{\mathrm{or}}}{1+\mathrm{W}_{3 \mathrm{r}}-\mathrm{W}_{4 \mathrm{r}}}\right)^{\frac{1+\mathrm{W}_{3 \mathrm{r}}-\mathrm{W}_{4 \mathrm{r}}}{2-\beta}}\left(\frac{(2-\beta) \mathrm{C}_{\mathrm{hr}} \mathrm{E}\left(\mathrm{D}_{\mathrm{r}}\right)}{2\left(1-\beta-\mathrm{W}_{3 \mathrm{r}}+\mathrm{W}_{4 \mathrm{r}}\right)}\right)^{\frac{1-\beta-\mathrm{W}_{3 \mathrm{r}}+\mathrm{W}_{4 \mathrm{r}}}{2-\beta}} \\
& \times\left(\frac{\mathrm{C}_{\mathrm{hr}} \mathrm{E}\left(\mathrm{D}_{\mathrm{r}}\right)}{2 \mathrm{~K}_{1} \mathrm{~W}_{3 \mathrm{r}}}\right)^{\mathrm{W}_{3 \mathrm{r}}}\left(\frac{\mathrm{C}_{\mathrm{hr}} \mathrm{E}\left(\mathrm{x}_{\mathrm{r}}\right) \mathrm{v}}{\mathrm{K}_{2} \mathrm{~W}_{4 \mathrm{r}}}\right)^{\mathrm{W}_{4 \mathrm{r}}}
\end{aligned}
$$

Taking the logarithm of both sides of (7):

$$
\begin{aligned}
\operatorname{lng}\left(\mathrm{W}_{3 \mathrm{r}}, \mathrm{W}_{4 \mathrm{r}}\right)= & \sum_{\mathrm{r}=1}^{\mathrm{n}}\left[\frac{1}{2-\beta}\left[1+\mathrm{W}_{3 \mathrm{r}}-\mathrm{W}_{4 \mathrm{r}}\right]\left\{\ln (2-\beta) \mathrm{C}_{\mathrm{or}}-\ln \left(1+\mathrm{W}_{3 \mathrm{r}}-\mathrm{W}_{4 \mathrm{r}}\right)\right\}\right. \\
& +\frac{1}{2-\beta}\left[1-\beta-\mathrm{W}_{3 \mathrm{r}}+\mathrm{W}_{4 \mathrm{r}}\right]\left\{\ln \frac{\mathrm{C}_{\mathrm{hr}} \mathrm{E}\left(\mathrm{D}_{\mathrm{r}}\right)(2-\beta)}{2}-\ln \left[1-\beta-\mathrm{W}_{3 \mathrm{r}}+\mathrm{W}_{4 \mathrm{r}}\right]\right\} \\
& \left.+\mathrm{W}_{3 \mathrm{r}}\left\{\ln \frac{\mathrm{C}_{\mathrm{hr}} \mathrm{E}\left(\mathrm{D}_{\mathrm{r}}\right)}{2 \mathrm{~K}_{1}}-\ln \mathrm{W}_{3 \mathrm{r}}\right\}+\mathrm{W}_{4 \mathrm{r}}\left\{\ln \frac{\mathrm{C}_{\mathrm{hr}} \mathrm{E}\left(\mathrm{x}_{\mathrm{r}}\right) v}{\mathrm{~K}_{2}}-\ln \mathrm{W}_{4 \mathrm{r}}\right\}\right]
\end{aligned}
$$

To calculate $\mathrm{W}_{3 \mathrm{r}}^{*}$ and $\mathrm{W}_{4 \mathrm{r}}^{*}$ which maximize $\mathrm{g}\left(\mathrm{W}_{3 \mathrm{r}}, \mathrm{W}_{4 \mathrm{r}}\right)$, equate the first partial derivatives of $\ln \mathrm{g}\left(\mathrm{W}_{3 \mathrm{r}}, \mathrm{W}_{4 \mathrm{r}}\right) \quad$ with respect to $\mathrm{W}_{3 \mathrm{r}}^{*}$ and $\mathrm{W}_{4 \mathrm{r}}^{*}$ respectively to zero as follows:

$$
\begin{aligned}
\frac{\partial \ln g\left(\mathrm{~W}_{3 \mathrm{r}}, \mathrm{W}_{4 \mathrm{r}}\right)}{\partial \mathrm{W}_{3 \mathrm{r}}} & =\frac{1}{2-\beta}\left\{\ln (2-\beta) \mathrm{C}_{\mathrm{or}}-\ln \left(1+\mathrm{W}_{3 \mathrm{r}}-\mathrm{W}_{4 \mathrm{r}}\right)\right\}-\frac{1}{2-\beta} \\
& -\frac{1}{2-\beta}\left\{\ln \frac{\mathrm{C}_{\mathrm{hr}} \mathrm{E}\left(\mathrm{D}_{\mathrm{r}}\right)(2-\beta)}{2}-\ln \left[1-\beta-\mathrm{W}_{3 \mathrm{r}}+\mathrm{W}_{4 \mathrm{r}}\right]\right\} \\
& +\frac{1}{2-\beta}+\left\{\ln \frac{\mathrm{C}_{\mathrm{hr}} \mathrm{E}\left(\mathrm{D}_{\mathrm{r}}\right)}{2 \mathrm{~K}_{1}}-\ln \mathrm{W}_{3 \mathrm{r}}\right\}-1=0
\end{aligned}
$$

Similarly:

$$
\begin{aligned}
\frac{\partial \operatorname{lng}\left(\mathrm{W}_{3 \mathrm{r}}, \mathrm{W}_{4 \mathrm{r}}\right)}{\partial \mathrm{W}_{4 \mathrm{r}}} & =\frac{-1}{2-\beta}\left\{\ln (2-\beta) \mathrm{C}_{\mathrm{or}}-\ln \left(1+\mathrm{W}_{3 \mathrm{r}}-\mathrm{W}_{4 \mathrm{r}}\right)\right\}+\frac{1}{2-\beta} \\
& +\frac{1}{2-\beta}\left\{\ln \frac{\mathrm{C}_{\mathrm{hr}} \mathrm{E}\left(\mathrm{D}_{\mathrm{r}}\right)(2-\beta)}{2}-\ln \left(1-\beta-\mathrm{W}_{3 \mathrm{r}}+\mathrm{W}_{4 \mathrm{r}}\right)\right\} \\
& -\frac{1}{2-\beta}+\left\{\ln \frac{\mathrm{C}_{\mathrm{hr}} \mathrm{E}\left(\mathrm{x}_{\mathrm{r}}\right) \mathcal{v}}{\mathrm{K}_{2}}-\ln \mathrm{W}_{4 \mathrm{r}}\right\}-1=0
\end{aligned}
$$

Simplifying the equation (8) and (9) and multiplying them, we get:

$\mathrm{W}_{3 \mathrm{r}} \mathrm{W}_{4 \mathrm{r}}=\left(\frac{\mathrm{C}_{\mathrm{hr}}^{2} \mathrm{E}\left(\mathrm{D}_{\mathrm{r}}\right) \mathrm{E}\left(\mathrm{x}_{\mathrm{r}}\right) \mathrm{v}}{2 \mathrm{~K}_{1} \mathrm{~K}_{2} \mathrm{e}^{2}}\right)$

Then, we obtain:

$$
\begin{aligned}
\mathrm{f}_{\mathrm{j}}\left(\mathrm{W}_{\mathrm{jr}}\right) & =\mathrm{W}_{\mathrm{jr}}^{4-\beta}+\mathrm{a}_{\mathrm{j}} \mathrm{W}_{\mathrm{jr}}^{3-\beta}-\mathrm{A}_{\mathrm{r}} \mathrm{W}_{\mathrm{jr}}^{2-\beta} \\
& +\mathrm{b}_{\mathrm{r}} \mathrm{W}_{\mathrm{jr}}^{2}-\mathrm{d}_{\mathrm{j}} \mathrm{W}_{\mathrm{jr}}-\mathrm{A}_{\mathrm{r}} \mathrm{b}_{\mathrm{r}}=0
\end{aligned}
$$

Where:

$$
\begin{aligned}
A_{r} & =\frac{C_{h r}^{2} E\left(D_{r}\right) E\left(x_{r}\right) v}{2 K_{1} K_{2} e^{2}}, \\
B_{r} & =\left(\frac{2 C_{o r}}{C_{h r} E\left(D_{r}\right)}\right)\left(\frac{C_{h r} E\left(D_{r}\right)}{2 K_{1} e}\right)^{2-\beta}, \\
C_{r} & =\left(\frac{C_{h r} E\left(D_{r}\right) v}{K_{2} e}\right)^{2-\beta}\left(\frac{C_{h r} E\left(D_{r}\right)}{2 C_{o r}}\right) \\
a_{j} & =\left\{\begin{array}{ll}
1, j=3 \\
1-\beta, j=4
\end{array},\right. \\
b_{j} & =\left\{\begin{array}{ll}
B_{r}, j=3 \\
C_{r}, j=4
\end{array}\right. \text { and } \\
d_{j} & = \begin{cases}B_{r}(1-\beta), j=3 \\
C_{r} & , j=4\end{cases}
\end{aligned}
$$

It could be easily proved that $\mathrm{fj}(0)<0$ and $\mathrm{fj}(1)>0, \forall \mathrm{j}=3,4$ and this is means that there exists a root $\mathrm{W}_{\mathrm{jr}} \varepsilon(0,1), \mathrm{j}=3,4$. Any method such as the trial and error, could be used to calculate these roots.

Now to verify that any root $\mathrm{W}_{3 \mathrm{r}}^{*}$ and $\mathrm{W}_{4 \mathrm{r}}^{*}$ calculated from equations (11) maximize $\mathrm{g}\left(\mathrm{W}_{3 \mathrm{r}}^{*}, \mathrm{~W}_{4 \mathrm{r}}^{*}\right)$ respectively. Applying the following conditions:

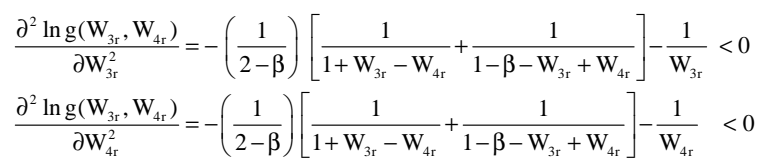

And: 
$\frac{\partial^{2} \ln g\left(\mathrm{~W}_{3 \mathrm{r}}, \mathrm{W}_{4 \mathrm{r}}\right)}{\partial \mathrm{W}_{3 \mathrm{r}} \partial \mathrm{W}_{4 \mathrm{r}}}=\left(\frac{1}{2-\beta}\right)\left[\frac{1}{1+\mathrm{W}_{3 \mathrm{r}}-\mathrm{W}_{4 \mathrm{r}}}+\frac{1}{1-\beta-\mathrm{W}_{3 \mathrm{r}}+\mathrm{W}_{4 \mathrm{r}}}\right]>0$

Hence:

$$
\begin{aligned}
\Delta & =\left(\frac{\partial^{2} \operatorname{lng}\left(\mathrm{W}_{3 \mathrm{r}}, \mathrm{W}_{4 \mathrm{r}}\right)}{\partial \mathrm{W}_{3 \mathrm{r}} \partial \mathrm{W}_{4 \mathrm{r}}}\right)^{2}-\left(\frac{\partial^{2} \operatorname{lng}\left(\mathrm{W}_{3 \mathrm{r}}, \mathrm{W}_{4 \mathrm{r}}\right)}{\partial \mathrm{W}_{3 \mathrm{r}}^{2}}\right)\left(\frac{\partial^{2} \operatorname{lng}\left(\mathrm{W}_{3 \mathrm{r}}, \mathrm{W}_{4 \mathrm{r}}\right)}{\partial \mathrm{W}_{4 \mathrm{r}}^{2}}\right) \\
& =-\left(\frac{1}{2-\beta}\right)\left[\frac{1}{1+\mathrm{W}_{3 \mathrm{r}}-\mathrm{W}_{4 \mathrm{r}}}+\frac{1}{1-\beta-\mathrm{W}_{3 \mathrm{r}}+\mathrm{W}_{4 \mathrm{r}}}\right]\left[\frac{1}{\mathrm{~W}_{3 \mathrm{r}}}+\frac{1}{\mathrm{~W}_{4 \mathrm{r}}}\right]-\frac{1}{\mathrm{~W}_{3 \mathrm{r}} \mathrm{W}_{4 \mathrm{r}}}<0
\end{aligned}
$$

Thus, the roots $\mathrm{W}_{3 \mathrm{r}}^{*}$ and $\mathrm{W}_{4 \mathrm{r}}^{*}$ calculated from equations (11) maximize the dual function $\mathrm{g}\left(\mathrm{W}_{3 \mathrm{r}}, \mathrm{W}_{4 \mathrm{r}}\right)$.

Hence the optimal solution is $\mathrm{W}_{\mathrm{jr}}^{*}, \mathrm{j}=1,2,3,4$, where $\mathrm{W}_{3 \mathrm{r}}^{*}$, are the solution of (11) and $\mathrm{W}_{1 \mathrm{r}}^{*}$, are calculated by substituting the values of $\mathrm{W}_{3 \mathrm{r}}^{*}$, in expression (6).

To find the optimal number of periods $\mathrm{N}_{\mathrm{r}}^{*}$, use the following relations due to Duffin and Peterson's theorem $^{[2]}$ as follows:

$$
\mathrm{C}_{\text {or }} \mathrm{N}_{\mathrm{r}}^{\beta-1}=\mathrm{W}_{1 \mathrm{r}}^{*} \mathrm{~g}\left(\mathrm{~W}_{3 \mathrm{r}}^{*}, \mathrm{~W}_{4 \mathrm{r}}^{*}\right)
$$

And:

$$
\frac{\mathrm{C}_{\mathrm{hr}} \mathrm{E}\left(\mathrm{D}_{\mathrm{r}}\right) \mathrm{N}_{\mathrm{r}}}{2}=\mathrm{W}_{2 \mathrm{r}}^{*} \mathrm{~g}\left(\mathrm{~W}_{3 \mathrm{r}}^{*}, \mathrm{~W}_{4 \mathrm{r}}^{*}\right)
$$

Solving these equations, the optimal expected number of periods per cycle is given by:

$$
N_{r}^{*}=\left(\frac{C_{h r} E\left(D_{r}\right)\left\{1+W_{3 r}^{*}-W_{4 r}^{*}\right\}}{2 C_{o r}\left\{1-\beta-W_{3 r}^{*}+W_{4 r}^{*}\right\}}\right)^{\frac{1}{\beta-2}}
$$

Then:

$$
Q_{m r}^{*}=\left(\frac{C_{h r}\left(E\left(D_{r}\right)\right)^{\beta-1}\left\{1+W_{3 r}^{*}-W_{4 r}^{*}\right\}}{2 C_{\text {or }}\left\{1-\beta-W_{3 r}^{*}+W_{4 r}^{*}\right\}}\right)^{\frac{1}{\beta-2}}+E\left(D_{r}\right) v
$$

\begin{tabular}{|c|c|c|c|c|}
\hline \multicolumn{2}{|c|}{$\begin{array}{l}\text { Items } \\
\text { Parameters }\end{array}$} & Item 1 & Item 2 & Item 3 \\
\hline $\begin{array}{l}\mathrm{E}\left(\mathrm{D}_{\mathrm{r}}\right) \\
\mathrm{C}_{\mathrm{hr}} \\
\mathrm{C}_{\mathrm{or}} \\
\mathrm{C}_{\mathrm{pr}}\end{array}$ & $\begin{array}{l}32 \\
0.20 \\
150 \\
100\end{array}$ & & $\begin{array}{l}25 \\
0.22 \\
170 \\
120\end{array}$ & $\begin{array}{l}18 \\
0.24 \\
190 \\
140\end{array}$ \\
\hline \multicolumn{5}{|c|}{$\begin{array}{l}\text { Also assuming that } v=5, K_{1}=10000, K_{2}=2000 \text { and } 0.5 \leq \beta<2 \\
\text { Solution: }\end{array}$} \\
\hline$\beta$ & $\mathrm{N}_{1}^{*}$ & $\mathrm{~N}_{2}^{*}$ & $\mathrm{~N}_{3}^{*}$ & $\min \mathrm{E}(\mathrm{TC})$ \\
\hline 0.5 & 6.24579 & 8.03712 & 10.609 & 9948.41 \\
\hline 0.6 & 6.07309 & 7.95435 & 10.7069 & 9997.24 \\
\hline 0.7 & 5.59853 & 7.48192 & 10.2982 & 10057.45 \\
\hline 0.8 & 4.62653 & 6.32425 & 8.9277 & 10129.85 \\
\hline 0.9 & 2.88917 & 4.03324 & 5.83927 & 10181.71 \\
\hline 1.0 & 0.540794 & 0.621639 & 0.683955 & 10245.53 \\
\hline 1.1 & 0.086965 & 0.0796744 & 0.0655885 & 10101.93 \\
\hline 1.2 & 0.0439282 & 0.0400897 & 0.0329991 & 9950.02 \\
\hline 1.3 & 0.0293183 & 0.0267516 & 0.0220258 & 9837.02 \\
\hline 1.4 & 0.0219954 & 0.0200696 & 0.016526 & 9760.91 \\
\hline 1.5 & 0.017598 & 0.0160574 & 0.0132227 & 9711.94 \\
\hline 1.6 & 0.0146656 & 0.0160574 & 0.0110196 & 9680.6 \\
\hline 1.7 & 0.0125721 & 0.0114709 & 0.00944583 & 9663.24 \\
\hline 1.8 & 0.0110049 & 0.0100395 & 0.0082663 & 9651.54 \\
\hline 1.9 & 0.00979254 & 0.00893033 & 0.00735091 & 9646.06 \\
\hline
\end{tabular}

Substituting the value of $N_{r}^{*}$ in equation (3) after adding the constant terms, we get:

$$
\begin{aligned}
\min \mathrm{E}(\mathrm{TC}) & =\sum_{\mathrm{r}=1}^{\mathrm{n}}\left[\mathrm{C}_{\mathrm{pr}} \mathrm{E}\left(\mathrm{D}_{\mathrm{r}}\right)+\mathrm{C}_{\mathrm{or}}\left(\frac{\mathrm{C}_{\mathrm{hr}} \mathrm{C}_{\mathrm{or}} \mathrm{E}\left(\mathrm{D}_{\mathrm{r}}\right)\left\{1+\mathrm{W}_{3 \mathrm{r}}^{*}-\mathrm{W}_{4 \mathrm{r}}^{*}\right\}}{2\left\{1-\beta-\mathrm{W}_{3 \mathrm{r}}^{*}+\mathrm{W}_{4 \mathrm{r}}^{*}\right.}\right)^{\frac{\beta-1}{\beta-2}}\right. \\
& \left.+\left(\frac{\mathrm{C}_{\mathrm{hr}} \mathrm{E}\left(\mathrm{D}_{\mathrm{r}}\right)}{2}\right)\left(\frac{\mathrm{C}_{\mathrm{hr}} \mathrm{E}\left(\mathrm{D}_{\mathrm{r}}\right)\left\{1+\mathrm{W}_{3 \mathrm{r}}^{*}-\mathrm{W}_{4 \mathrm{r}}^{*}\right\}}{2 \mathrm{C}_{\mathrm{or}}\left\{1-\beta-\mathrm{W}_{3 \mathrm{r}}^{*}+\mathrm{W}_{4 \mathrm{r}}^{*}\right\}}\right)^{\frac{1}{\beta-2}}+\mathrm{C}_{\mathrm{hr}} \mathrm{E}\left(\mathrm{D}_{\mathrm{r}}\right) v\right]
\end{aligned}
$$

Table 1: The parameters of three items

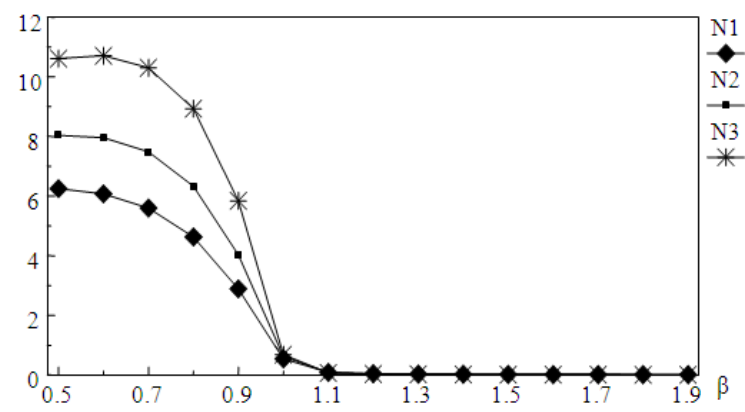

Fig. 2: The Relation between $N_{r}^{*}$ and $\beta$

Special case: Let $\beta=0, r=1$ and $\mathrm{K}_{1}, \mathrm{~K}_{2} \rightarrow \infty \Rightarrow \mathrm{C}_{\mathrm{Or}}\left(\mathrm{N}_{\mathrm{r}}\right)$ $=\mathrm{C}_{0}=$ constant, $\mathrm{W}_{3 \mathrm{r}}^{*}, \mathrm{~W}_{4 \mathrm{r}}^{*} \rightarrow 0$ and $\mathrm{W}_{1 \mathrm{r}}^{*}=\mathrm{W}_{2 \mathrm{r}}^{*}=1 / 2$. This is a probabilistic single-item inventory model without any restriction and constant costs, which agree with the model of maintaining stock to absorb demand fluctuations $^{[3]}$, the equations (12), (13) and (14) become:

$$
N^{*}=\sqrt{\frac{2 C_{o}}{C_{h} E(D)}}, Q_{m}^{*}=\sqrt{\frac{2 C_{o} E(D)}{C_{h}}}+E\left(D_{r}\right) v
$$

And:

$\min E(T C)=C_{p} E(D)+\sqrt{2 C_{h} C_{o} E(D)}+C_{h r} E\left(D_{r}\right) v$ 


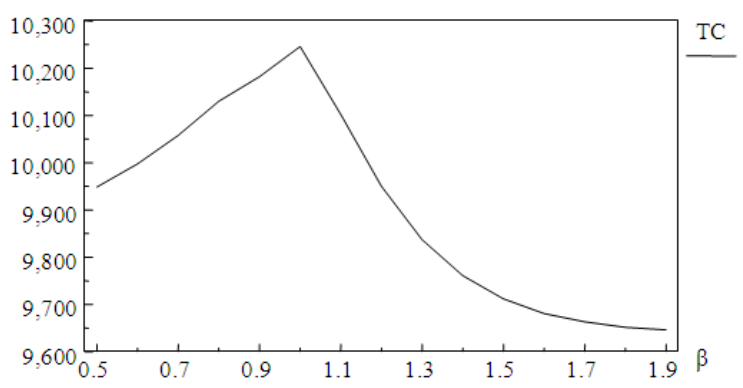

Fig. 3: The Relation between mine (TC) and $\beta$

An illustrative example: Let us find the optimal expected number of periods and the minimum expected total cost min E (TC) for the previous model of periodic review probabilistic multi-item inventory system with zero lead time under constraints and varying order cost, on the data of Table 1.

Also, by using the freelance program we can draw the relation between ${ }^{*}$, min $E$ (TC) against $\beta$ as shown in Fig. 2 and 3 respectively.

$$
\begin{aligned}
& \mathrm{N}_{\mathrm{r}}^{*} \\
& \text { Min E (TC) }
\end{aligned}
$$

\section{CONCLUSION}

We have evaluated the optimal expected number of periods $\mathrm{N}_{\mathrm{r}}^{*}, \mathrm{r}=1,2, \ldots, \mathrm{n}$, then we deduced the minimum expected total cost min $\mathrm{E}$ (TC) of the considered safety stock probabilistic multi-item inventory model. We draw the curves and min E (TC) against $\beta$, which indicate the values of $\mathrm{N}_{\mathrm{r}}^{*}$ and $\beta$ that give the minimum value of the expected total cost of our numerical example.

\section{REFERENCES}

1. Abou-El-Ata, Fergany, H.A. and M.F. El-Wakeel, 2002. Probabilistic multi-item inventory model with varying order cost under two restrictions: A geometric programming approach. Intl. J. Product. Econ., 83: 223-231.

2. Duffin, R.J. and E.L. Peterson, 1974. Constrained minima treated by geometric means. Westinghouse Scientific paper, 64: 158-129.

3. Fabrycky, W.J. and J. Banks, 1967. Procurement and Inventory Systems: Theory and Analysis. Reinhold Publishing Corporation, USA.

4. Hadley, G. and T.M. Whitin, 1963. Analysis of Inventory Systems. Englewood Cliffs, N.J. Prentice-Hall.

5. Hariri, A.M.A. and M.O. Abou-El-Ata, 1995. Multi-item production lot-size inventory model with varying order cost under a restriction: A Geometric programming approach. Product. Plan. Control., 6: 374-377.

6. Taha, H.A., 1997. Operations Research. 6th Edn. Prentice-Hall, INC, Englewood Cliffs, NJ, USA. 